\title{
A CHARACTERIZATION OF BI-LIPSCHITZ EMBEDDABLE METRIC SPACES IN TERMS OF LOCAL BI-LIPSCHITZ EMBEDDABILITY
}

\author{
JEEHYEON SEO
}

\begin{abstract}
We characterize uniformly perfect, complete, doubling metric spaces, which embed bi-Lipschitzly into Euclidean space. Our result applies in particular to spaces of Grushin type equipped with Carnot-Carathéodory distance. Hence we obtain the first example of a sub-Riemannian manifold admitting such a bi-Lipschitz embedding. Our techniques involve a passage from local to global information, building on work of Christ and McShane. A new feature of our proof is the verification of the co-Lipschitz condition. This verification splits into a large-scale case and a local case. These cases are distinguished by a relative distance map which is associated to a Whitney-type decomposition of an open subset $\Omega$ of the space. We prove that if the Whitney cubes embed uniformly bi-Lipschitzly into a fixed Euclidean space, and if the complement of $\Omega$ also embeds, then so does the full space.
\end{abstract}

\section{Introduction}

A map between two metric spaces is bi-Lipschitz if distances in the image and source should not exceed distances in the source and image respectively by more than a fixed, universal multiplicative constant. More precisely, a map $f$ between metric spaces $\left(X, d_{X}\right)$ and $\left(Y, d_{Y}\right)$ is called bi-Lipschitz if there exists an $L \geq 1$ such that

$$
\frac{1}{L} d_{X}(x, y) \leq d_{Y}(f(x), f(y)) \leq L d_{X}(x, y)
$$

for all $x, y \in X$.

Bi-Lipschitz maps play a role in computer science as well as in many branches of mathematics. Solving the Sparsest cut problem approximately is important in the theory of approximation algorithms. The best-known algorithm for this question is related to the Goemans-Linial conjecture [9, 15]. Recently, Cheeger and Kleiner [7] together with Lee and Naor [20] gave an counterexample to the Goemans-Linial conjecture. They showed that the Heisenberg group admits a metric which is of negative type, yet does not admit a bi-Lipschitz embedding into $L^{1}$.

Bi-Lipschitz maps are related to problems of differentiability by Rademacher's theorem. Lipschitz maps form the right substitute for smooth maps in the theory of analysis on metric spaces. We would like to know for which metric spaces the resulting analysis is genuinely new and for which ones the analysis can be seen as just classical analysis on a suitable subset of a Banach space. This leads to the question

Received by the editors xxx.

1991 Mathematics Subject Classification. Primary: 30L05; Secondary: 53C17.

Key words and phrases. Bi-Lipschitz embedding; Uniformly perfect; Whitney decomposition; The Grushin Plane; Coloring map. 
to characterize metric spaces that embed bi-Lipschitzly into classical Banach spaces. However, the characterization of metric spaces which are bi-Lipschitz equivalent to $\left(\mathbb{R}^{n}, E u c l\right)$ or even of metric spaces, which are bi-Lipschitzly embeddable into $\left(\mathbb{R}^{n}\right.$, Eucl) remain difficult open problems in Geometric Analysis.

We are interested in the question of which metric spaces embed bi-Lipschitzly into Euclidean space. We state some progress on this problem. Assouad gave a partial answer: every snowflaked version of a doubling metric space embeds bi-Lipschitzly into some Euclidean space [1]. Even though the theorem of Assouad completely answers the question, which metric spaces are quasisymmetrically embeddable into Euclidean space, this result does not guarantee bi-Lipschitz embeddability of the original metric space. In particular, the Heisenberg group, which is a doubling metric space, admits no bi-Lipschitz embedding into Euclidean space. Luosto [16] together with Luukkainen and Movahedi-Lankarani [17] gave a precise relationship between Assouad dimension and dimension of receiving Euclidean space for ultra metric spaces: an ultrametric space is bi-Lipschitzly embeddable into $\mathbb{R}^{n}$ if and only if its Assouad dimension is less than $n$.

Semmes [22] showed that $\mathbb{R}^{n}$ equipped with any metric $\delta_{\omega}$ deformed by $A_{1}$-weight $\omega$ admits a bi-Lipschitz embedding into some $\mathbb{R}^{N}$. However, $\left(\mathbb{R}^{n}, \delta_{\omega}\right)$ may be not bi-Lipschitzly equivalent to $\mathbb{R}^{n}$. Bishop [3] constructed a Sierpinski carpet $E \subset \mathbb{R}^{2}$ and an $A_{1}$-weight $\omega$ which blows up on $E$. In this construction, he showed that $w$ is not comparable to the Jacobian of any quasiconformal mapping.

In this paper, we will characterize uniformly perfect complete metric spaces which admit a bi-Lipschitz embedding in terms of uniform local bi-Lipschitz embeddability. Indeed, uniform perfectness and existence of a doubling measure yield existence of a Whitney-type decomposition. Furthermore, uniform local bi-Lipschitz embeddability of Christ cubes associated with such a decomposition implies global bi-Lipschitz embeddability.

Theorem 1.1. A uniformly perfect complete metric space $(X, d)$ admits a bi-Lipschitz embedding into some Euclidean space if and only if the following conditions hold:

(1) it supports a doubling measure $\mu$,

(2) there exists a closed subset $Y$ of $X$ which admits a bi-Lipschitz embedding into some $\mathbb{R}^{M_{1}}$,

(3) $\Omega=X \backslash Y$ admits uniformly Christ-local bi-Lipschitz embeddings into some $\mathbb{R}^{M_{2}}$.

The bi-Lipschitz constant and dimension of receiving Euclidean space depend on the data of the metric space $X$, the doubling constant of $\mu, M_{1}, M_{2}$, and the bi-Lipschitz constants in conditions (2) and (3).

We now discuss applications of Theorem 1.1 to the bi-Lipschitz embedding question for sub-Riemannian manifolds. For more information of Carnot-Carathéodory geometry, see [10] and [19]. Pansu [21] showed that a version of Rademacher's differentiation theorem holds for Lipschitz maps on Carnot groups: every Lipschitz map between Carnot groups is almost everywhere differentiable in some sense and its differential is a Lie group homomorphism. Semmes observed that Pansu's result implies that nonabelian Carnot groups admit no bi-Lipschitz embedding into Euclidean space (Theorem 7.1 in [23]). Cheeger proved a remarkable extension of Rademacher's theorem 
for doubling $p$-Poincaré spaces and gave a corresponding nonembedding theorem (see Section 10 and Theorem 14.3 in [4]).

By Cheeger's theorem, we can deduce nonembeddability of certain regular subRiemannian manifolds. However, his result does not apply to singular sub-Riemannian manifolds. This paper is motivated by the question whether or not the Grushin plane embeds bi-Lipschitzly into Euclidean space. While the Grushin plane is one of the simplest singular sub-Riemannian manifold, the previous known nonembedding theorems do not apply. In contrast, as an application of Theorem 1.1 we will prove bi-Lipschitz embeddability of the Grushin plane. This is the first example of a sub-Riemannian manifold that embeds bi-Lipschitzly into Euclidean space.

Definition 1.1. The Grushin plane $\mathbb{G}$ is $\mathbb{R}^{2}$ with horizontal distribution spanned by

$$
X_{1}=\frac{\partial}{\partial x} \quad \text { and } \quad X_{2}=x \frac{\partial}{\partial y} .
$$

Theorem 1.2. The Grushin plane equipped with Carnot-Carathéodory distance admits a bi-Lipschitz embedding into some Euclidean space.

The structure of this paper follows. In the second section, we shall see Assouad's embedding theorem and Lipschitz extension theorem. We will review Michael Christ's construction of a system of dyadic cubes [8] in doubling metric spaces. We will next construct a Whitney-type decomposition, which we call a Christ-Whitney decomposition (Lemma 2.1) for a uniformly perfect space supporting a doubling measure. We will also introduce some definitions and lemmas which set the stage for Theorem 1.1.

In the following section, we shall characterize bi-Lipschitz embeddable metric spaces by proving Theorem 1.1. To this end, we first apply McShane's extension theorem to extend a Lipschitz map on $Y$ to $X$. We introduce the Whitney distance map $d_{\mathrm{W}}$ (Definition 2.7). It is the key tool for construction of a co-Lipschitz map. We break the Christ-Whitney decomposition into two parts using the Whitney distance map. After some basic preliminaries, we will construct a W-local co-Lipschitz and W-large scale co-Lipschitz map on these parts (Lemmas 3.1 and 3.2).

In Section 3, we discuss applications of Theorem 1.1 to the bi-Lipschitz embedding question for sub-Riemannian manifolds. We prove bi-Lipschitz embeddability of the Grushin plane into some Euclidean space.

\section{Preliminaries}

2.1. Notation and terminology. For a metric space $X=(X, d)$, we write $\operatorname{diam}(A)$ (or $\operatorname{diam}_{d}(A)$ in case we need to mention the metric) for the diameter of a set $A \subset X$, and $\operatorname{dist}(A, B)$ for the distance between nonempty sets $A, B \subset X$. We abbreviate $\operatorname{dist}(A, x)=\operatorname{dist}(A,\{x\})$ for a set $A \subset X$ and $x \in X$. We abbreviate $R^{n}$ for $\left(R^{n}\right.$, Eucl) and write $d_{E}$ for the Euclidean metric. As customary, we let $C, c, \cdots$ denote finite positive constants. These constants may depend on auxiliary data $a$, $b$, etc ; we indicate this by writing $C(a, b)$ or $c(a, b)$. We also write $a \lesssim b$ if there is a constant $C$ such that $a \leq C b$.

We recall that the map $f: X \rightarrow Y$ is a bi-Lipschitz embedding if (1.1) holds. We do not assume that $f$ is onto. We say an invertible map $f: X \rightarrow Y$ between metric spaces is co-Lipschitz if $f^{-1}$ is Lipschitz. We call any constant $L$ satisfying (1.1) a bi-Lipschitz constant for $f$. 
Definition 2.1. A metric space $(X, d)$ is uniformly perfect if there exists a constant $A>0$ such that for each $x \in X$ and $0<r<\operatorname{diam} X$ there is a point $y \in X$ which satisfies $A^{-1} r \leq d(x, y) \leq r$. We say that $(X, d)$ is $A$-uniformly perfect.

Uniform perfectness implies nonexistence of separating annuli of large modulus and nonexistence of isolated points. Every connected metric space is uniformly perfect. In an $A$-uniformly perfect space, $\bar{B}(x, r) \backslash B\left(x, A^{-1} r\right)$ is nonempty for all $x \in X$ and $0<r<\operatorname{diam} X$ and so $A^{-1} r \leq \operatorname{diam} B(x, r) \leq 2 r$.

The doubling condition provides a kind of boundedness of the geometry of the space.

Definition 2.2. A Borel measure $\mu$ in a metric space is called doubling if balls have finite and positive measure for any nonempty ball and there is a constant $D \geq 1$ such that

$$
\mu(B(x, 2 r)) \leq D \mu(B(x, r))
$$

for all $x \in X$ and $r>0$. We call $D$ a doubling constant.

Definition 2.3. A metric space is called doubling if there is a constant $C$ so that every set of diameter $d$ in the space can be covered by at most $C$ sets of diameter at most $d / 2$.

2.2. Basic theorems. In this section, we recall Assouad's embedding theorem and McShane's Lipschitz extension theorem.

Definition 2.4 (Snowflaking). If $(X, d)$ is a metric space, then its snowflaking is a metric space $\left(X, d^{\epsilon}\right)$, where $0<\epsilon<1$. We say that $\left(X, d^{\epsilon}\right)$ is a snowflaked version of $(X, d)$.

Theorem 2.1 (Assouad [1]). Each snowflaked version of a doubling metric space admits a bi-Lipschitz embedding into some Euclidean space. If $0<\epsilon<1$, then $\left(\mathbb{R}, d_{E}{ }^{\epsilon}\right)$ embeds bi-Lipschitzly into $\mathbb{R}^{k}$, where $k$ is the smallest integer which is greater than $\frac{1}{\epsilon}$.

The identity snowflaking $(X, d) \rightarrow\left(X, d^{\epsilon}\right)$ is $t^{\epsilon}$-quasisymmetric and hence, each metric space is quasisymmetrically embedded in Euclidean space if and only if it is doubling. However, Assouad's theorem does not answer whether or not the original metric space embeds bi-Lipschitzly. For example, whereas the snowflaking of the Heisenberg group endowed with Carnot-Carathéodory distance, $\left(\mathbb{H}, d_{c c}{ }^{\epsilon}\right)$, admits a bi-Lipschitz embedding into some Euclidean space, the Heisenberg group is not bi-Lipschitzly embeddable into any Euclidean space. Such nonembeddability is a consequence of Pansu's Rademacher-type theorem [21] as observed by Semmes [23]. It also follows from Cheeger's nonembedding theorem [4].

To prove Theorem 2.1, Assouad builds a multiscale family of maps on scale $2^{-j}$ for each $j \in \mathbb{Z}$ and glues these maps together into an embedding using $2^{-j}$-nets and a coloring map. A similar idea will appear in the proof of Theorem 3.1. In fact, we shall consider a Whitney-type decomposition instead of nets and use a coloring map to increase dimension of receiving Euclidean space.

With some restrictions on $X$ and $Y$, and for $A \subset X$, every Lipschitz function $f: A \rightarrow Y$ can be extended to a Lipschitz function $F: X \rightarrow Y$. We recall McShane's 
Lipschitz extension theorem. Since McShane's Lipschitz extension map has no restriction on the source space, it is useful for our purpose. For further information, see $[12,13]$.

Theorem 2.2 (McShane). Let $X$ be an arbitrary metric space. If $A \subset X$ and $f: A \rightarrow \mathbb{R}$ is L-Lipschitz, then there exists an L-Lipschitz function $F: X \rightarrow \mathbb{R}$ which extends f. i.e. $\left.F\right|_{A}=f$.

Corollary 2.1 (McShane). Let $f: A \rightarrow \mathbb{R}^{M}$ where $A \subset X$, be an L-Lipschitz function. Then, there exists an $\sqrt{M}$ L-Lipschitz function $F: X \rightarrow \mathbb{R}^{M}$ such that $\left.F\right|_{A}=f$.

2.3. Christ-Whitney decomposition. As Euclidean space has a system of dyadic cubes, every doubling metric measure space also has a system of sets akin to classical dyadic cubes. The following Proposition 2.1 may be transparent if we think of $Q_{\alpha}^{k}$ as being essentially a cube of diameter roughly $\delta^{k}$ with center $z_{\alpha}^{k}$. When $Q_{\beta}^{k+1} \subset Q_{\alpha}^{k}$, we say that $Q_{\beta}^{k+1}$ is a child of $Q_{\alpha}^{k}$ and $Q_{\alpha}^{k}$ is a parent of $Q_{\beta}^{k+1}$.

Proposition 2.1 (Christ [8]). Let $(X, d, \mu)$ be a doubling metric measure space. Then, there exists a collection of open subsets $\left\{Q_{\alpha}^{k} \subset X \mid k \in \mathbb{Z}, \alpha \in I_{k}\right\}$ where $I_{k}$ is some index set depending on $k$, and constants $\delta \in(0,1), a_{0} \in(0,1), \eta>0$ and $C_{1}, c<\infty$ such that

(1) $\mu\left(X \backslash \cup_{\alpha \in I_{k}} Q_{\alpha}^{k}\right)=0, \quad$ for all $k \in \mathbb{Z}$.

(2) For any $\alpha, \beta, k$, and $l$ with $l \geq k$, either $Q_{\beta}^{l} \subset Q_{\alpha}^{k}$ or $Q_{\beta}^{l} \bigcap Q_{\alpha}^{k}=\emptyset$.

(3) Each $Q_{\alpha}^{k}$ has exactly one parent and at least one child for all $k \in \mathbb{Z}$.

(4) For each $(\alpha, k)$, there exists $z_{\alpha}^{k} \in X$ such that

$$
B\left(z_{\alpha}^{k}, a_{0} \delta^{k}\right) \subset Q_{\alpha}^{k} \subset B\left(z_{\alpha}^{k}, C_{1} \delta^{k}\right) .
$$

We now introduce a Whitney-type decomposition on an open subset of a uniformly perfect metric space supporting a doubling measure. As open subset of Euclidean space has a Whitney decomposition from a system of dyadic cubes, we have a Whitney-type decomposition from a system of Christ cubes. We call it a ChristWhitney decomposition. This decomposition has a comparability condition (see (4) Lemma 2.1) in addition to all conditions of a Whitney decomposition. This comparability condition together with doubling condition will play an important role in the proof of Lemma 2.3, which yields existence of a coloring map in Lemma 2.4.

Lemma 2.1. Suppose that $(X, d, \mu)$ is a A-uniformly perfect metric space supporting a doubling metric measure, $Y$ is a closed subset of $X$, and $\Omega=X \backslash Y$. Then $\Omega$ has a Christ-Whitney decomposition $\mathrm{M}_{\Omega}$ satisfying the following properties:

(1) $\mu\left(\Omega \backslash \cup_{Q \in \mathrm{M}_{\Omega}} Q\right)=0$.

(2) $\operatorname{diam}(Q) \leq \operatorname{dist}(Q, Y) \leq \frac{4 C_{1} A}{\delta} \operatorname{diam}(Q)$.

(3) $Q \cap Q^{\prime}=\emptyset$.

(4) For any $Q \in \mathrm{M}_{\Omega}$, there exists $x \in \Omega$ such that

$$
(*) B\left(x, a_{0} \delta^{k}\right) \subset Q \subset B\left(x, C_{1} \delta^{k}\right)
$$

for some $k$.

The constants $\delta, a_{0}$ and $C_{1}$ are deduced from Proposition 2.1. 
Remark 2.1. We say that $Q$ is $\left(C_{1}, a_{0}\right)$-quasiball if $(*)$ holds for some $x$ and $\delta$. From now on, we will call a ball $B\left(x, C_{1} \delta^{k}\right)$ containing $Q$ a $C_{1}$-quasiball of $Q$ and denote it by $\widetilde{B_{Q}}$. We observe that $\operatorname{diam}\left(\widetilde{B_{Q}}\right)$ is comparable to $\delta^{k}$ by uniform perfectness of $X$.

Proof. Since $\Omega=X \backslash Y$ is a doubling metric measure space, we have a family of subsets

$$
\left\{Q_{\alpha}^{k} \subset \Omega \mid k \in \mathbb{Z}, \alpha \in I_{k}\right\}
$$

for fixed constants $\delta$ and $C_{1}$ so that $\mu\left(\Omega \backslash \cup_{\alpha \in I_{k}} Q_{\alpha}^{k}\right)=0$ from Proposition 2.1. We now consider layers, defined by $\Omega_{k}=\left\{x \mid c^{\prime} \delta^{k}<\operatorname{dist}(x, Y) \leq c^{\prime} \delta^{k-1}\right\}$, where $c^{\prime}$ is a positive constant we shall fix momentarily. Obviously, $\Omega=\cup_{k=-\infty}^{\infty} \Omega_{k}$.

We now make an initial choice of $Q$ 's, and denote the resulting collection by $\mathrm{M}_{0}$. Our choice is made as follows. We consider $Q$ 's chosen from $\mathcal{A}^{k}=\left\{Q_{\alpha}^{k} \mid \alpha \in I_{k}\right\}$ for each $k \in \mathbb{Z}$, (each such $Q$ is of size approximately $\delta^{k}$ ), and include a $Q$ in $\mathrm{M}_{0}$ if it intersects $\Omega_{k}$. In other words,

$$
\mathrm{M}_{0}=\cup_{k}\left\{Q \in \mathcal{A}^{k} \mid Q \cap \Omega_{k} \neq \emptyset\right\}
$$

We then have $\mu\left(\Omega \backslash \cup_{Q \in \mathrm{M}_{0}} Q\right)=0$. For an appropriate choice of $c^{\prime}$,

$$
\operatorname{diam}(Q) \leq \operatorname{dist}(Q, Y) \leq \frac{4 C_{1} A}{\delta} \operatorname{diam}(Q)
$$

Let us prove (2.2) first. Suppose $Q \in \mathcal{A}^{k}$, then $\frac{1}{A} \delta^{k} \leq \operatorname{diam}(Q) \leq 2 C_{1} \delta^{k}$ because of uniform perfectness. Since $Q \in \mathrm{M}_{0}$, there exists $x \in Q \cap \Omega_{k}$. Thus, $\operatorname{dist}(Q, Y) \leq$ $\operatorname{dist}(x, Y) \leq c^{\prime} \delta^{k-1} \leq \frac{c^{\prime} A}{\delta} \operatorname{diam} Q \leq \frac{4 C_{1} A}{\delta} \operatorname{diam}(Q)$ and $\operatorname{dist}(Q, Y) \geq \operatorname{dist}(x, Y)-$ $\operatorname{diam}(Q) \geq c^{\prime} \delta^{k}-2 C_{1} \delta^{k}=2 C_{1} \delta^{k} \geq \operatorname{diam}(Q)$. If we choose $c^{\prime}=4 C_{1}$, we get the equation (2.2).

Notice that the collection $\mathrm{M}_{0}$ has all required properties, except that $Q$ 's in it are not necessarily disjoint. To finish the proof of the lemma we need to refine our choice leading to $\mathrm{M}_{0}$, eliminating $Q$ 's which were really unnecessary. We require the following observation. Suppose $Q \in \mathcal{A}^{k}$ and $Q^{\prime} \in \mathcal{A}^{k^{\prime}}$. If $Q$ and $Q^{\prime}$ are not disjoint, then one of two must be contained in the other. Start now with any $Q \in \mathrm{M}_{0}$, and consider the unique maximal parent in $\mathrm{M}_{0}$ which contains it. We let $\mathrm{M}_{\Omega}$ denote the collection of maximal $Q$ 's in $\mathrm{M}_{0}$. The last property comes straightforward from Proposition 2.1 and Lemma 2.1 is therefore proved.

We now define new concepts $Q^{*}$ and $Q^{* *}$ corresponding to a Whitney cube $Q$ and a dilated Whitney cube $\lambda Q$ respectively in the classical Whitney decomposition.

Definition 2.5. For any fixed $Q \in \mathrm{M}_{\Omega}$, we denote by $Q^{*}$ the collection of all $R \in \mathrm{M}_{\Omega}$ whose distance from $Q$ does not exceed minimum diameters of $R$ and $Q$ by a fixed constant $\epsilon$. We denote by $Q^{* *}$ the collection of all $S \in \mathrm{M}_{\Omega}$ whose distance from some $R \in Q^{*}$ does not exceed minimum diameters of $R$ and $S$ by a fixed constant $\epsilon$. 

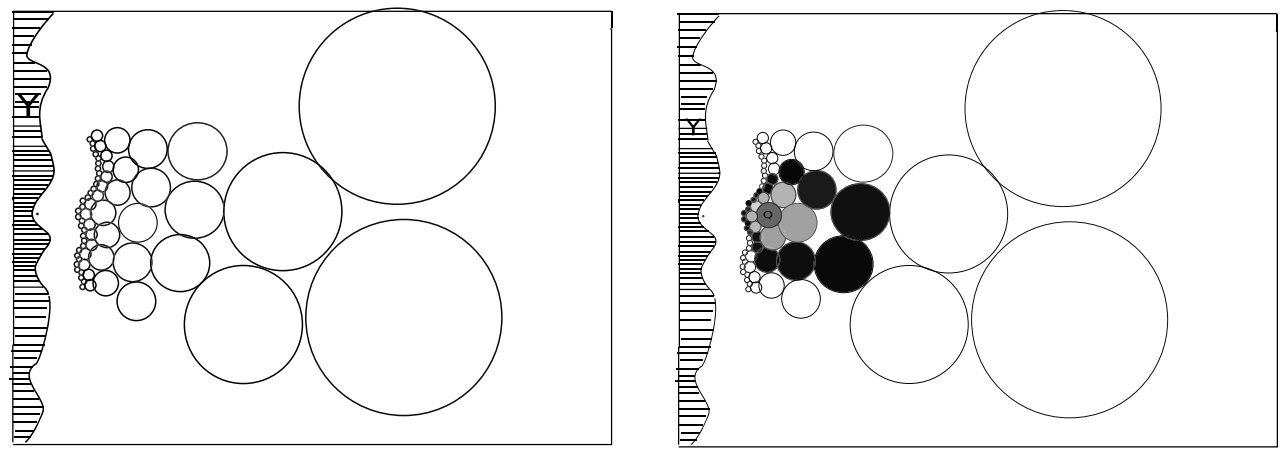

Figure 1. The gray balls are elements of $Q^{*}$ and gray and black balls are elements of $Q^{* *}$.
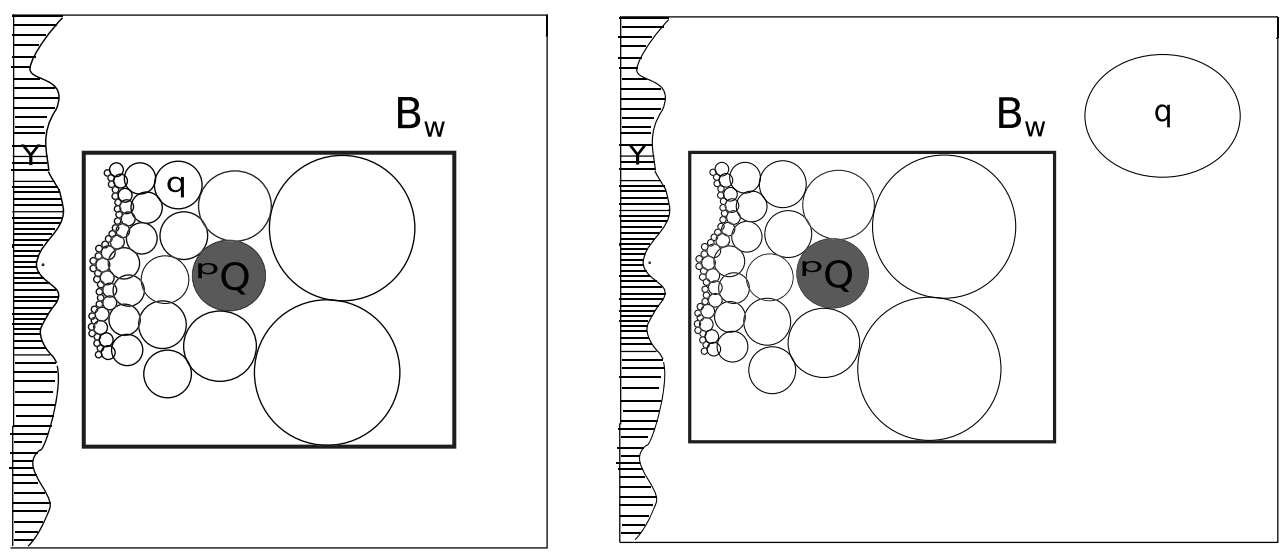

FiguRE 2. Let the square be the Whitney distance ball of radius $16 M_{1} L_{1}^{2}$ centered at $Q$. W-local co-Lipschitz means $|f(p)-f(q)| \gtrsim$ $d(p, q)$ for any $p \in Q$ and $q \in R$ where $d_{\mathrm{W}}(Q, R)<16 M_{1} L_{1}^{2}$. Wlarge scale co-Lipschitz means $|f(p)-f(q)| \gtrsim d(p, q)$ for $p \in Q$ and $q \in R$ with $d_{\mathrm{W}}(Q, R) \geq 16 M_{1} L_{1}^{2}$.

Here $\epsilon$ is a fixed number such that $0<\epsilon<1$. In other words,

(1) $Q^{*}=\cup\left\{R \in \mathrm{M}_{\Omega} \mid \operatorname{dist}(Q, R)<\epsilon \min \{\operatorname{diam}(Q), \operatorname{diam}(R)\}\right\}$.

(2) $Q^{* *}=\cup\left\{S \in \mathrm{M}_{\Omega} \mid \operatorname{dist}(S, R)<\epsilon \min \{\operatorname{diam}(S)\right.$, $\operatorname{diam}(R)\}$ for some $R \in$ $\left.Q^{*}\right\}$.

Remark 2.2. $Q^{*}$ could contain no other Christ-cubes except $Q$. Throughout this paper, we can choose any $\epsilon$. However, in practice, we will restrict $\epsilon$ to a universal fixed number in $(0,1)$ since we will consider condition of uniformly Christ-local bi-Lipschitz embeddings (Definition 2.6).

Remark 2.3. Figures 1-3 illustrate an idea how our construction goes. Of course, actual shapes will depend on a metric space.

We next see some propositions related to $Q^{*}$ and $Q^{* *}$. 

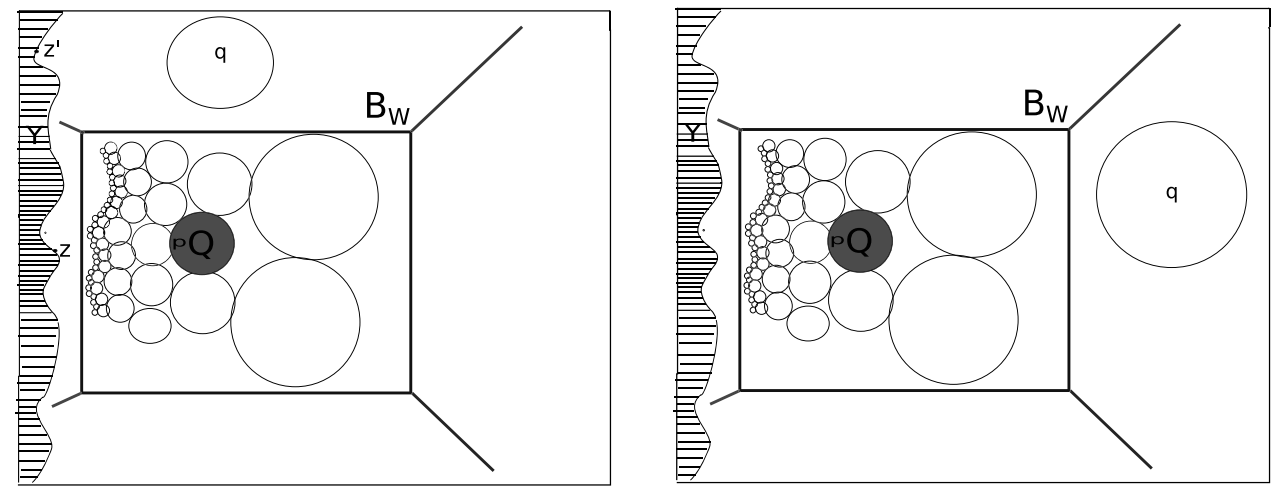

Figure 3. $g$ and dist $(\cdot, Y)$ satisfy W-large scale co-Lipschitz bounds.

Proposition 2.2. For any fixed $Q \in \mathrm{M}_{\Omega}$, suppose $R \in Q^{*}$. Then,

$$
\left[\frac{4 C_{1} A}{\delta}+1+\epsilon\right]^{-1} \operatorname{diam}(R) \leq \operatorname{diam}(Q) \leq\left[\frac{4 C_{1} A}{\delta}+1+\epsilon\right] \operatorname{diam}(R)
$$

Proof. We suppose that $\operatorname{diam}(R) \geq \operatorname{diam}(Q)$. Then, we arrive at

$$
\begin{aligned}
\operatorname{diam}(R) & \leq \operatorname{dist}(R, Y) \\
& \leq \operatorname{diam}(Q)+\operatorname{dist}(Q, Y)+\operatorname{dist}(R, Q) \\
& \leq\left[\frac{4 C_{1} A}{\delta}+1+\epsilon\right] \operatorname{diam}(Q)
\end{aligned}
$$

and the symmetrical implication proves the proposition.

Proposition 2.3. Let $(X, d)$ be a uniformly perfect metric space supporting a doubling measure $\mu$, and let $\mathrm{M}_{\Omega}$ be a Christ-Whitney decomposition as in Lemma 2.1.

(1) Suppose $Q \in \mathrm{M}_{\Omega}$. Then there are at most $N$ Christ cubes in $\mathrm{M}_{\Omega}$ in $Q^{* *}$.

(2) Any point in $\mathrm{M}_{\Omega}$ is contained in at most $N$ of $Q^{* *}$.

The number $N$ is independent of $Q$. It depends on the doubling constant of $\mu, \epsilon$, and the data of $X$.

Proof. For any $R \in Q^{* *}$, we have comparability between $\operatorname{diam}(Q)$ and $\operatorname{diam}(R)$ from Proposition 2.2. Therefore, $\operatorname{diam}\left(Q^{* *}\right)$ is comparable to $\operatorname{diam}(Q)$. Doubling condition yields that there are at most a finite number of such $R$ 's and hence there are at most $N\left(\mu, C_{1}, A, \delta, \epsilon\right)$ Christ cubes in $Q^{* *}$.

Let $p$ be a point in $\mathrm{M}_{\Omega}$ and write $p \in R$. We now observe that for any $Q \in R^{* *}$, we have $R \in Q^{* *}$. We have $p \in Q^{* *}$ for all $Q \in R^{* *}$ and hence $p$ is contained in at most $N$ sets of type $Q^{* *}$ by Proposition $2.3(1)$.

We now build a family of Lipschitz cutoff functions. We will use these functions to construct a W-local co-Lipschitz map by composing with uniformly Christ-local bi-Lipschitz embeddings. See Lemma 3.2. 
Lemma 2.2. There exist functions $\varphi_{Q}: X \rightarrow \mathbb{R}$ where $Q \in \mathrm{M}_{\Omega}$ with the following properties:

(1) $0 \leq \varphi_{Q} \leq 1$,

(2) $\left.\varphi_{Q}\right|_{Q^{*}}=1$,

(3) $\left.\varphi_{Q}\right|_{X \backslash Q^{* *}}=0$,

(4) $\varphi_{Q}$ is Lipschitz with constant $\frac{C}{\operatorname{diam}(Q)}$,

(5) For all $p \in \Omega$, we have $\varphi_{Q}(p) \neq 0$ for at most $N$ cubes $Q \in \mathrm{M}_{\Omega}$.

Here, $C$ and $N$ denote uniformly fixed constants independent of the choice of element $Q \in \mathrm{M}_{\Omega}$. They depend on the data of $X, \epsilon$, and the doubling constant of $\mu$.

Proof. We define

$$
\varphi_{Q}(x)=\min \left\{1, \quad \frac{\operatorname{dist}\left(x, X \backslash Q^{* *}\right)}{\operatorname{dist}\left(Q^{*}, X \backslash Q^{* *}\right)}\right\} .
$$

Then, (1), (2), and (3) are obvious and (5) follows from Proposition 2.3. To check (4), note that

$$
\left|\varphi_{Q}(p)-\varphi_{Q}(q)\right| \leq \frac{d(p, q)}{\operatorname{dist}\left(Q^{*}, X \backslash Q^{* *}\right)}
$$

Thus, it suffices to show that

$$
\operatorname{dist}\left(Q^{*}, X \backslash Q^{* *}\right) \geq c \operatorname{diam}(Q)
$$

To this end, let $x$ be a point in $Q^{*}$. We write $x \in R$ for some $R \in Q^{*}$ and choose $y \in S \in X \backslash Q^{* *}$. Then,

$$
\begin{aligned}
d(x, y) & \geq \operatorname{dist}(R, S) \\
& \geq \epsilon \min \{\operatorname{diam}(R), \operatorname{diam}(S)\} \\
& \geq C\left(L_{1}, A, \delta, \epsilon\right) \operatorname{diam}(Q)
\end{aligned}
$$

The last inequality is deduced from the comparability between $\operatorname{diam}(R)$ and $\operatorname{diam}(Q)$ in case $\operatorname{diam}(S) \geq \operatorname{diam}(R)$. Otherwise, $\operatorname{diam}(R) \geq \operatorname{diam}(S)$, we divide into two cases, either

$$
\text { (1) } \operatorname{diam}(R) \geq \operatorname{diam}(S) \geq \frac{1}{2\left[\frac{4 C_{1} A}{\delta}+1\right]} \operatorname{diam}(R)
$$

or

$$
(2) \operatorname{diam}(S)<\frac{1}{2\left[\frac{4 C_{1} A}{\delta}+1\right]} \operatorname{diam}(R) \text {. }
$$


In the first case, we have obviously comparability between $\operatorname{diam}(S)$ and $\operatorname{diam}(R)$. In the second case, we use the comparability condition of a Christ-Whitney decomposition. Then,

$$
\begin{aligned}
\operatorname{dist}(R, S) & \geq \operatorname{dist}(R, Y)-\operatorname{dist}(S, Y)-\operatorname{diam}(S) \\
& \geq \operatorname{diam}(R)-\left[\frac{4 C_{1} A}{\delta}+1\right] \operatorname{diam}(S) \\
& \geq \frac{1}{2} \operatorname{diam}(R) \\
& \geq C\left(L_{1}, A, \delta\right) \operatorname{diam}(Q) .
\end{aligned}
$$

Therefore, the proof of (4) is completed.

Remark 2.4. We use the fact that $\varphi_{Q}=1$ on $Q^{*}$ and $\varphi_{Q}=0$ off $Q^{* *}$ so that the map $\widetilde{h}_{Q}=h_{Q} \cdot \varphi_{Q}$ defined in Subsection 3.3 is bi-Lipschitz on $Q^{*}$ and supported on $Q^{* *}$. These properties are needed in the proof of Lemma 3.2; see case (3).

Definition 2.6. Let $(X, d, \mu)$ be a uniformly perfect metric space supporting a doubling measure and let $Y$ be a closed subset of $X$. We say that $\Omega=X \backslash Y$ admits uniformly Christ-local bi-Lipschitz embeddings if there exist bi-Lipschitz embeddings of each $Q^{* *}$ into a fixed Euclidean space with uniform bi-Lipschitz constant.

2.4. Whitney distance map. The following relative distance map plays a key role to construct a co-Lipschitz map from a metric space into Euclidean space in Section 3. We will break $\mathrm{M}_{\Omega}$ into two parts and construct co-Lipschitz maps on these parts (Definition 3.1) by using the Whitney distance map.

Definition 2.7. The Whitney distance map $d_{\mathrm{W}}$ on $\mathrm{M}_{\Omega} \times \mathrm{M}_{\Omega}$ is defined by

$$
d_{\mathrm{W}}(Q, R)=\frac{\operatorname{dist}(Q, R)}{\min (\operatorname{diam}(Q), \operatorname{diam}(R))} .
$$

Remark 2.5. The Whitney distance map $d_{\mathrm{W}}$ is not a metric. In fact, if $\bar{Q} \cap \bar{R} \neq \emptyset$, then $d_{\mathrm{W}}(Q, R)=0$. We observe that

$$
d_{\mathrm{W}}(Q, R) \leq d_{\mathrm{W}}(Q, S)+d_{\mathrm{W}}(S, R)+1
$$

if $\operatorname{diam}(S) \leq \min \{\operatorname{diam}(Q), \operatorname{diam}(R)\}$.

Throughout this paper, we will use the terminology Whitney distance ball of radius $\rho$ for the set of all elements in $M_{\Omega}$ such that Whitney distance to a fixed center cube in $\mathrm{M}_{\Omega}$ is less than $\rho$. We write $B_{\mathrm{W}}(Q, \rho)$ for the Whitney distance ball of radius $\rho$ with center $Q$.

The next lemma allows us to construct a coloring map that gives different colors to Christ cubes within a given Whitney distance ball.

Lemma 2.3. Each Whitney distance ball of radius $\rho$ contains a finite number of elements of the Christ-Whitney decomposition $\mathrm{M}_{\Omega}$. The number depends on the doubling constant of $\mu$ and $\rho$.

Proof. We fix a Christ cube $Q \in \mathrm{M}_{\Omega}$ and we require to count the number of $R \in \mathrm{M}_{\Omega}$ such that $d_{\mathrm{W}}(Q, R)<\rho$. We have two cases either $(1) \operatorname{diam}(Q)<\operatorname{diam}(R)$ or $(2)$ $\operatorname{diam}(R) \leq \operatorname{diam}(Q)$. 
Suppose $\operatorname{diam}(Q)<\operatorname{diam}(R)$. Then, we have

$$
\operatorname{dist}(R, Y)-\operatorname{dist}(Q, Y)<\operatorname{dist}(Q, R)+\operatorname{diam}(Q)<(\rho+1) \operatorname{diam}(Q) .
$$

Since $\operatorname{dist}(Q, Y) \leq \frac{4 C_{1} A}{\delta} \operatorname{diam}(Q)$, we have an upper bound for $\operatorname{diam}(R)$ in terms of $\operatorname{diam}(Q)$. That is, $\operatorname{diam}(R)<\left(\rho+1+\frac{4 C_{1} A}{\delta}\right) \operatorname{diam}(Q)$.

Similarly, $\operatorname{diam}(R)$ has a lower bound in terms of the size of $Q$ in the case of $\operatorname{diam}(R) \leq \operatorname{diam}(Q)$ :

$$
\operatorname{diam}(R) \geq\left(\rho+1+\frac{4 C_{1} A}{\delta}\right)^{-1} \operatorname{diam}(Q) .
$$

Therefore, the number of $R \in \mathrm{M}_{\Omega}$ in $B_{\mathrm{W}}(Q, \rho)$ is the sum of the cardinality of the following sets:

$$
\left\{R \in \mathrm{M}_{\Omega} \mid \begin{array}{c}
\operatorname{diam}(Q)<\operatorname{diam}(R)<\left(\rho+1+\frac{4 C_{1} A}{\delta}\right) \operatorname{diam}(Q), \\
\operatorname{dist}(Q, R)<\rho \operatorname{diam}(Q)
\end{array}\right\}
$$

and

$$
\left\{R \in \mathrm{M}_{\Omega} \mid \begin{array}{c}
\operatorname{diam}(R) \leq \operatorname{diam}(Q)<\left(\rho+1+\frac{4 C_{1} A}{\delta}\right) \operatorname{diam}(R), \\
\operatorname{dist}(Q, R)<\rho \operatorname{diam}(R)
\end{array}\right\}
$$

Now we suppose that $p$ and $q$ are centers of $C_{1}$-quasiballs $\widetilde{B_{Q}}$ and $\widetilde{B_{R}}$ which have approximately sizes of $Q$ and $R$. If $R$ is in either the set (2.3) or the set (2.4), then we find that

$$
d(p, q) \leq \operatorname{diam}(Q)+\operatorname{dist}(Q, R)+\operatorname{diam}(R)<\left(2 \rho+1+\frac{4 C_{1} A}{\delta}\right) \operatorname{diam}(Q)
$$

Thus, the number of $R \in \mathrm{M}_{\Omega}$ in $B_{\mathrm{W}}(Q, \rho)$ is at most twice of the number of centers $q$ satisfying (2.5). In other words, we can count the number of $R$ 's in (2.3) and (2.4) by counting the number of centers of $C_{1}$-quasiballs $\widetilde{B_{R}}$. By the doubling condition, the ball centered at $p$ with radius $\left(2 \rho+1+\frac{4 C_{1} A}{\delta}\right) \operatorname{diam}(Q)$ can be covered by finite number of $C_{1}$-quasiballs centered at such $q$. Finally, the comparability of the size of $R$ and that of the ball centered at $q$ concludes Lemma 2.3.

We write the number of Christ cubes within Whitney distance ball of radius $\rho$ as $m=m(\rho, D)$ in terms of $\rho$ and the doubling constant $D$ of $\mu$.

Lemma 2.4. There exists a coloring map

$$
K: \mathrm{M}_{\Omega} \longrightarrow\{1,2,3, \ldots, M\} \text { for some } M>m(m-1)
$$

such that any two boxes within Whitney distance ball of radius $\rho$ have different colors. In other words, if $R^{\prime}, R^{\prime \prime}$ have $d_{\mathrm{W}}\left(R^{\prime}, R^{\prime \prime}\right)<\rho$, then $K\left(R^{\prime}\right) \neq K\left(R^{\prime \prime}\right)$.

Proof. We apply Zorn's lemma. Let us consider the partially ordered set $(\mathcal{P}, \leqslant)$ where $\mathcal{P}$ is the collection of maps $k$ defined from $\mathcal{S} \subset \mathrm{M}_{\Omega}$ to $\{1,2, \ldots, M\}$ so that $k(R) \neq k\left(R^{\prime}\right)$ for all $R, R^{\prime} \in \mathcal{S}$ whose Whitney distance is less than $\rho$. The inequality $(k, \mathcal{S}) \leqslant\left(k^{\prime}, \mathcal{S}^{\prime}\right)$ means $k^{\prime}$ is a extension of $k\left(\mathcal{S} \subset \mathcal{S}^{\prime} \in \mathcal{P}\right.$ and $\left.\left.k^{\prime}\right|_{\mathcal{S}}=k\right)$.

By Zorn's lemma, there exists a maximal element $\widehat{k}$. If the domain of $\widehat{k}$ is $\mathrm{M}_{\Omega}$, then we can set $K=\widehat{k}$. Otherwise, take $Q^{\prime} \in \mathrm{M}_{\Omega} \backslash$ domain $(\widehat{k})$. We now want to give a color to $Q^{\prime}$. The color of $Q^{\prime}$ should differ from any color already assigned to any $R$ 
where $d_{\mathrm{W}}\left(Q^{\prime}, R\right)<\rho$ and also differ from any color already assigned to any $S$ where $d_{\mathrm{W}}(S, R)<\rho$ and $d_{\mathrm{W}}\left(Q^{\prime}, R\right)<\rho$. We observe that the number of such $R$ is at most $m-1$ and the number of $S$ for given $R$ is at most $m$. Thus, the total number of colors seen is at most $m(m-1)$. Since $M>m(m-1)$, there is a color remaining which can be assigned to $Q^{\prime}$; this contradicts maximality of $\widehat{k}$.

\section{Bi-Lipschitz embeddable metric spaces}

Now we are ready to state the main theorem. It asserts that in a uniformly perfect complete metric space supporting a doubling measure, the local information of uniformly Christ-local bi-Lipschitz embeddability (Definition 2.6) can be turned into global information of bi-Lipschitz embeddability.

Theorem 3.1. A uniformly perfect complete metric space $(X, d)$ admits a bi-Lipschitz embedding into some Euclidean space if and only if the following conditions hold:

(1) it supports a doubling measure $\mu$,

(2) there exists a closed subset $Y$ of $X$ which admits a bi-Lipschitz embedding into some $\mathbb{R}^{M_{1}}$,

(3) $\Omega=X \backslash Y$ admits uniformly Christ-local bi-Lipschitz embeddings into some $\mathbb{R}^{M_{2}}$.

The bi-Lipschitz constant and dimension of receiving Euclidean space depend on the data of the metric space $X$, the doubling constant of $\mu, M_{1}, M_{2}$, and the bi-Lipschitz constants in conditions (2) and (3).

\section{Outline of proof}

Suppose that we have a $L$-bi-Lipschitz embedding $f$ from $(X, d)$ into $\mathbb{R}^{n}$ for some $n$. Euclidean space is a doubling metric space and the doubling condition is bi-Lipschitz invariant. Hence, $(X, d)$ is a complete doubling metric space. Thus, there exists a doubling measure $\mu([18,24])$. The second condition is trivial, setting $Y=X$. The third condition is trivial since $\Omega=\emptyset$.

The content of the theorem is the other implication: a uniformly perfect complete space satisfying (1), (2), and (3) embeds bi-Lipschitzly in some Euclidean space. We will use Proposition 3.1 to complete the main theorem. Since the full measure set $\mathrm{M}_{\Omega} \cup Y$ is dense in $X$ and the constructed map in Proposition 3.1 is uniformly continuous, Theorem 3.1 follows immediately. Therefore, we will focus on proving Proposition 3.1 in Subsections 3.1, 3.2, and 3.3.

Proposition 3.1. Let $(X, d, \mu)$ be a A-uniformly perfect, complete, doubling metric space and let $Y$ be a closed subset of $X$. Then, the full measure set $M_{\Omega} \cup Y$ admits a bi-Lipschitz embedding into some Euclidean space if the followings are satisfied:

(1) $Y$ admits a bi-Lipschitz embedding into some $\mathbb{R}^{M_{1}}$,

(2) $\Omega=X \backslash Y$ admits uniformly Christ-local bi-Lipschitz embeddings into some $\mathbb{R}^{M_{2}}$. The bi-Lipschitz constant and dimension of receiving Euclidean space depend on the data of metric space $X$, the doubling constant, $M_{1}, M_{2}$, and the bi-Lipschitz constants in conditions (1) and (2).

We briefly outline the proof of Proposition 3.1. We first extend a (bi)-Lipschitz map $f$ on $Y$ to a global Lipschitz map $g$ on $X$, using McShane's extension theorem (see Theorem 2.2 and Corollary 2.1). We then suppose that $f$ is a $L_{1}$-bi-Lipschitz 
embedding from $Y$ into $\mathbb{R}^{M_{1}}$. From McShane's theorem, we have a $\sqrt{M_{1}} L_{1}$-Lipschitz extension map

$$
g: X \longrightarrow \mathbb{R}^{M_{1}} \quad \text { such that }\left.g\right|_{Y}=f .
$$

From now on we fix such $L_{1}$ and $M_{1}$ is chosen sufficiently large relative to other data $C_{1}, A$, and $\delta$. The precise choice of $M_{1}$ will be made in connection with the estimate in (3.1).

In general, the map $g$ is not globally co-Lipschitz on a full measure set $\mathrm{M}_{\Omega}$ of $\Omega$. Therefore, we next shall construct a co-Lipschitz map using a local and large scale argument in the sense of Whitney distance on a Christ-Whitney decomposition (see Definitions 2.7 and 3.1).

Definition 3.1. Let $Q$ be any fixed cube in $\mathrm{M}_{\Omega}$. We say $f: \mathrm{M}_{\Omega} \rightarrow \mathbb{R}^{n}$ is W-local co-Lipschitz if it is co-Lipschitz for any two points $p \in Q, q \in R$ where $R$ is in $B_{\mathrm{W}}\left(Q, 16 M_{1} L_{1}^{2}\right)$. We say $f$ is W-large scale co-Lipschitz if it is co-Lipschitz for any two points $p \in Q$ and $q \in R$ where $R$ is not in $B_{\mathrm{W}}\left(Q, 16 M_{1} L_{1}^{2}\right)$ (see Figure 2).

In Subsection 3.1, we will construct a W-large scale co-Lipschitz map and global Lipschitz map on $\mathrm{M}_{\Omega}$. To this end, we will break the complement of an arbitrary Whitney distance ball of radius $16 M_{1} L_{1}{ }^{2}$ into two parts using relative distance in terms of the distance between two cubes and their maximum diameter. We shall see that McShane's extension map $g$ and distance map from $Y$, dist $(\cdot, Y)$, which are global Lipschitz maps, are W-large scale co-Lipschitz on these two parts respectively.

In Subection 3.2, we will construct a W-local co-Lipschitz map on $\mathrm{M}_{\Omega}$ via putting together all local patches of bi-Lipschitz embeddings. We will assign different colors to elements in a Christ-Whitney decomposition within arbitrary Whitney distance ball of radius $16 M_{1} L_{1}^{2}$.

Finally, in Subection 3.3, we will construct a global bi-Lipschitz embedding on the full measure set $\mathrm{M}_{\Omega} \cup Y$ of $X$ completing the proof of Lemma 3.1.

3.1. W-large-scale co-Lipschitz and global Lipschitz map on $M_{\Omega}$. We construct a W-large scale co-Lipschitz and global Lipschitz map on a full measure set $\mathrm{M}_{\Omega} \subset \Omega$. Roughly speaking, McShane's extension map guarantees a W-large scale co-Lipschitz bound for points $p, q$ in $\mathrm{M}_{\Omega}$ whose distance is big enough with respect to the maximum diameter of cubes containing them. Whenever $p \in Q$ and $q \in R$ have the property that $\operatorname{dist}(Q, R)$ exceeds their maximum diameter by a fixed constant, we consider points $z, z^{\prime}$ in $Y$ which realize distances to $p, q$ respectively. Then, $|g(p)-g(z)|$ and $\left|g(q)-g\left(z^{\prime}\right)\right|$ are approximately greater than the maximum diameter and we can conclude co-Lipschitz from the triangle inequality. Furthermore, when the distance between two points is small enough with respect to the maximum diameter, $|d(p, Y)-d(q, Y)|$ is approximately greater than the maximum diameter (see Figure 3).

Lemma 3.1. Let $Q$ be any fixed cube in $\mathrm{M}_{\Omega}$. For any two points $p \in Q$ and $q \in R$, where $d_{\mathrm{W}}(Q, R) \geq 16 M_{1} L_{1}{ }^{2}$, the McShane extension map $g$ and $\operatorname{dist}(\cdot, Y)$ satisfy $\mathrm{W}$-large scale co-Lipschitz bounds. More precisely,

(1) If $\frac{\operatorname{dist}(Q, R)}{\max (\operatorname{diam}(Q), \operatorname{diam}(R))} \geq \frac{8 M_{1} L_{1}{ }^{2}}{1+\frac{4 C_{1} A}{\delta}}$, then $|g(p)-g(q)| \geq C\left(L_{1}, M_{1}\right) d(p, q)$. 
(2) If $\frac{\operatorname{dist}(Q, R)}{\max (\operatorname{diam}(Q), \operatorname{diam}(R))} \leq \frac{8 M_{1} L_{1}{ }^{2}}{1+\frac{4 C_{1} A}{\delta}}$, then $|\operatorname{dist}(p, Y)-\operatorname{dist}(q, Y)| \geq C\left(L_{1}, M_{1}\right) d(p, q)$.

Proof. We may assume that $\operatorname{diam}(R) \geq \operatorname{diam}(Q)$ without loss of generality. We choose $z, z^{\prime} \in Y$ such that $\operatorname{dist}(Y, Q)=\operatorname{dist}(z, Q)$ and $\operatorname{dist}(Y, R)=\operatorname{dist}\left(z^{\prime}, R\right)$. We claim that $z \neq z^{\prime}$. In fact, $d\left(z, z^{\prime}\right) \geq \frac{1}{2} d(p, q)$. To conclude the claim, we suppose that $d\left(z, z^{\prime}\right)<\frac{1}{2} d(p, q)$. Then,

$$
d(p, q) \leq d(p, z)+d\left(z, z^{\prime}\right)+d\left(z^{\prime}, q\right) .
$$

Thus, we have

$$
\begin{aligned}
d(p, q) & \leq 2\left[d(p, z)+d\left(z^{\prime}, q\right)\right] \\
& \leq 2\left[\operatorname{dist}(z, Q)+\operatorname{diam}(Q)+\operatorname{dist}\left(z^{\prime}, R\right)+\operatorname{diam}(R)\right] \\
& \leq 2[\operatorname{dist}(Y, Q)+\operatorname{diam}(Q)+\operatorname{dist}(Y, R)+\operatorname{diam}(R)] \\
& \leq 2\left(\frac{4 C_{1} A}{\delta}+1\right)[\operatorname{diam}(Q)+\operatorname{diam}(R)] \\
& \leq 2\left(\frac{4 C_{1} A}{\delta}+1\right)\left(\frac{1+\frac{4 C_{1} A}{\delta}}{8 M_{1} L_{1}{ }^{2}}+\frac{1}{16 M_{1} L_{1}{ }^{2}}\right) \operatorname{dist}(Q, R) \\
& \leq \frac{\left(1+\frac{4 C_{1} A}{\delta}\right)\left(3+\frac{4 C_{1} A}{\delta}\right)}{8 M_{1} L_{1}{ }^{2}} d(p, q) .
\end{aligned}
$$

This is a contradiction provided $M_{1}$ is selected sufficiently large relative to $C_{1}, A$, and $\delta$. Now,

$$
\begin{aligned}
|g(p)-g(q)| & \geq\left|f(z)-f\left(z^{\prime}\right)\right|-|f(z)-g(p)|-\left|f\left(z^{\prime}\right)-g(q)\right| \\
& \geq \frac{1}{L_{1}} d\left(z, z^{\prime}\right)-C d(z, p)-C d\left(z^{\prime}, q\right) .
\end{aligned}
$$

where $C=\sqrt{M_{1}} L_{1}$ from McShane's theorem. We have

$$
\begin{aligned}
d(p, z) & \leq\left(\frac{4 C_{1} A}{\delta}+1\right) \operatorname{diam}(Q) \leq \frac{\left(\frac{4 C_{1} A}{\delta}+1\right)}{16 M_{1} L_{1}{ }^{2}} \operatorname{dist}(Q, R) \\
& \leq \frac{\left(\frac{4 C_{1} A}{\delta}+1\right)}{16 M_{1} L_{1}{ }^{2}} d(p, q) .
\end{aligned}
$$

Similarly, we have

$$
\left|g(q)-g\left(z^{\prime}\right)\right| \leq L_{1} d\left(z^{\prime}, q\right) \leq \frac{\left(\frac{4 C_{1} A}{\delta}+1\right)^{2}}{8 M_{1} L_{1}^{2}} d(p, q) .
$$


In conclusion,

$$
\begin{aligned}
|g(p)-g(q)| & \geq\left[\frac{1}{2 L_{1}}-2 C \frac{\left(\frac{4 C_{1} A}{\delta}+1\right)^{2}}{8 M_{1} L_{1}^{2}}\right] d(p, q) \\
& \geq \frac{1}{2 L_{1}}\left[1-\frac{\left(\frac{4 C_{1} A}{\delta}+1\right)^{2}}{2 \sqrt{M_{1}}}\right] d(p, q) \\
& \geq \frac{1}{4 L_{1}} d(p, q)
\end{aligned}
$$

since we can choose $M_{1}$ sufficiently large. This completes the proof of the first case.

In second case, we have

$$
16 M_{1} L_{1}^{2} \operatorname{diam}(Q) \leq \operatorname{dist}(Q, R) \leq \frac{8 M_{1} L_{1}^{2}}{1+\frac{4 C_{1} A}{\delta}} \operatorname{diam}(R) .
$$

Therefore, $2\left(1+\frac{4 C_{1} A}{\delta}\right) \operatorname{diam}(Q) \leq \operatorname{diam}(R)$. We now have

$$
\begin{aligned}
|\operatorname{dist}(p Y)-\operatorname{dist}(q Y)| & \geq \operatorname{dist}(q, Y)-\operatorname{dist}(p, Y) \\
& \geq \operatorname{dist}(R, Y)-\operatorname{dist}(Q, Y)-\operatorname{diam}(Q) \\
& \geq \operatorname{diam}(R)-\left(1+\frac{4 C_{1} A}{\delta}\right) \operatorname{diam}(Q) \\
& \geq \frac{1}{2} \operatorname{diam}(R)
\end{aligned}
$$

while $d(p, q) \leq \operatorname{diam}(Q)+\operatorname{dist}(Q, R)+\operatorname{diam}(R) \lesssim \operatorname{diam}(R)$. Thus, we proved the second case.

3.2. W-local co-Lipschitz and global Lipschitz map on $\mathbf{M}_{\boldsymbol{\Omega}}$. We next construct a W-local co-Lipschitz and global Lipschitz map on a full measure set $\mathrm{M}_{\Omega} \subset \Omega$ into some Euclidean space. In general, $M_{1}+1$, the dimension of the target space of $g(\cdot) \times \operatorname{dist}(\cdot, Y)$ is not large enough to construct a co-Lipschitz map. Hence, we will use a coloring map that gives additional dimension of the Euclidean space (see Lemma 2.4).

Suppose that $h_{Q}$ 's are $L_{2}$-bi-Lipschitz embeddings of $Q^{* *}$ for each $Q \in \mathrm{M}_{\Omega}$ into $\mathbb{R}^{M_{2}}$ with uniformly determined $L_{2}$ and $M_{2}$. Now we consider the map

$$
\widetilde{h}_{Q}=h_{Q} \cdot \varphi_{Q}: X \longrightarrow \mathbb{R}^{M_{2}} ;
$$

it is bi-Lipschitz on $Q^{*}$, Lipschitz on $X$, and supported on $Q^{* *}$. We recall that $\left\{\varphi_{Q}\right\}$ is a family of Lipschitz cutoff functions as in Lemma 2.2. Then, we may assume that for some $c$

$$
\widetilde{h}_{Q}\left(Q^{*}\right) \subset B\left(0, c L_{2} \operatorname{diam}(Q)\right) \backslash B\left(0, \frac{1}{c L_{2}} \operatorname{diam}(Q)\right),
$$

because we can postcompose with an isometric translation map of $\mathbb{R}^{M_{2}}$ if necessary. Next, we will put together all patches to make a W-local co-Lipschitz map by assigning different colors to each element in $M_{\Omega}$. We will denote $\left\{e_{1}, e_{2}, \ldots, e_{M}\right\}$ by an orthonormal basis for $\mathbb{R}^{M}$. 
Lemma 3.2. The following map $H$ from $\mathrm{M}_{\Omega}$ into $\left(\mathbb{R}^{M_{2}}\right)^{M}$ given by

$$
H(p)=\sum_{Q \in \mathrm{M}_{\Omega}} \widetilde{h}_{Q}(p) \otimes e_{K(Q)},
$$

is a global Lipschitz and W-local co-Lipschitz map. The (W-local) bi-Lipschitz constant depends on $L_{1}, L_{2}$ and $M_{1}$. That is,

$$
|H(p)-H(q)| \geq C\left(L_{1}, L_{2}, M_{1}\right) d(p, q)
$$

for any points $p$ in any fixed $Q$ and $q$ in $R$ where $d_{\mathrm{W}}(Q, R)<16 M_{1} L_{1}{ }^{2}$.

Proof. Since $\widetilde{h}_{Q}$ is bi-Lipschitz on $Q^{*}$ with the uniform bi-Lipschitz constant $L_{2}$, Lipschitz on $X$, and supported on $Q^{* *}$, the map $H$ is a finite sum of Lipschitz maps from Proposition 2.3. Thus, it is Lipschitz on $\Omega$. Now, we will show that $H$ is a W-local co-Lipschitz map according to positions of two points $p$ and $q$ on $\mathrm{M}_{\Omega}$. There are three cases.

(1) If $p, q \in Q^{*}$, then $\widetilde{h}_{Q}$ is bi-Lipschitz on $Q^{*}$ and $Q$ is the element in $\mathrm{M}_{\Omega}$ that shares the same color at $p$ and $q$. Therefore, we find that

$$
\begin{aligned}
|H(p)-H(q)| & \geq\left|\widetilde{h}_{Q}(p)-\widetilde{h}_{Q}(q)\right| \\
& =\left|h_{Q}(p)-h_{Q}(q)\right|, \quad \text { since }\left.\varphi_{Q}\right|_{Q^{*}}=1 \\
& \geq \frac{1}{L_{2}} d(p, q)
\end{aligned}
$$

since $h_{Q}$ is $L_{2}$-bi-Lipschitz.

(2) If $p \in Q, q \notin Q^{* *}$, then $\widetilde{h}_{Q}(q)=0$. Thus, we have

$$
\begin{aligned}
|H(p)-H(q)| & \geq\left|\widetilde{h}_{Q}(p)-\widetilde{h}_{Q}(q)\right|=\left|\widetilde{h}_{Q}(p)\right| \\
& \geq \frac{1}{c L_{2}} \operatorname{diam}(Q) .
\end{aligned}
$$

On the other hand, we observe that

$$
\begin{aligned}
d(p, q) & \leq \operatorname{diam}(Q)+\operatorname{dist}(Q, R)+\operatorname{diam}(R) \\
& \leq \operatorname{diam}(Q)+\operatorname{dist}(Q, R)+\operatorname{dist}(R, Y) \\
& \leq 2 \operatorname{diam}(Q)+2 \operatorname{dist}(Q, R)+\operatorname{dist}(Q, Y)
\end{aligned}
$$

Since $\operatorname{dist}(Q, Y) \leq \frac{4 C_{1} A}{\delta} \operatorname{diam}(Q)$ and

$$
\operatorname{dist}(Q, R) \leq 16 M_{1} L_{1}^{2} \min \{\operatorname{diam}(Q), \operatorname{diam}(R)\} \leq 16 M_{1} L_{1}^{2} \operatorname{diam}(Q),
$$

we conclude

$$
d(p, q) \lesssim \operatorname{diam}(Q)
$$

and so $|H(p)-H(q)| \gtrsim d(p, q)$ as desired.

(3) If $p \in Q, q \in Q^{* *}$, then there is $R \in Q^{*}$ so that $p, q \in R^{*}$ and $\widetilde{h}_{R}$ is bi-Lipschitz on $R^{*}$. Therefore, we conclude the following from the first case:

$$
|H(p)-H(q)| \geq\left|\widetilde{h}_{R}(p)-\widetilde{h}_{R}(q)\right| \geq \frac{1}{L_{2}} d(p, q) .
$$


3.3. Global bi-Lipschitz embedding on a full measure set $\mathrm{M}_{\Omega} \cup Y$. Finally, we are ready to construct a global bi-Lipschitz embedding on a full measure set of $X$. We define the map $F$ from $\mathrm{M}_{\Omega} \cup Y$ into $\mathbb{R}^{M_{1}} \times\left(R^{M_{2}}\right)^{M} \times \mathbb{R}$ as follows:

$$
F(p)= \begin{cases}g(p) \times H(p) \times \operatorname{dist}(p, Y), & \text { for } p \in \mathrm{M}_{\Omega} \\ f(p) \times\{0\} \times\{0\}, & \text { for } p \in Y .\end{cases}
$$

Then $F$ is Lipschitz on a full measure set $\mathrm{M}_{\Omega} \subset \Omega$ because $g$ and $\operatorname{dist}(\cdot, Y)$ are Lipschitz on $X$ and $H$ is a finite sum of Lipschitz maps on $\mathrm{M}_{\Omega}$. Moreover, when we define $H(q)=0$ for $q \in Y$, then for every $p \in \mathrm{M}_{\Omega}$ and any $q \in Y$, we arrive at

$$
\begin{aligned}
|H(p)-H(q)|=|H(p)| & =\left|\sum_{Q \in \mathrm{M}_{\Omega}} \widetilde{h}_{Q}(p) \otimes e_{K(Q)}\right| \\
& \leq N L_{2} \operatorname{diam}(Q) \\
& \leq N L_{2} \operatorname{dist}(Q, Y) \\
& \leq N L_{2} d(p, q)
\end{aligned}
$$

We have shown that $F$ is co-Lipschitz on $\mathrm{M}_{\Omega}$ by Lemmas 3.1 and 3.2 and $\left.F\right|_{Y}=f$ is co-Lipschitz. Finally, we have a bi-Lipschitz embedding $F$ from a full measure set $\mathrm{M}_{\Omega} \cup Y$ of $X$ into $\mathbb{R}^{M_{1}} \times\left(R^{M_{2}}\right)^{M} \times \mathbb{R}$. The bi-Lipschitz constant depends on the data of metric space $X$, the doubling constant of $\mu, M_{1}, M_{2}, L_{1}$, and $L_{2}$. Therefore, Proposition 3.1 is proved.

\section{Applications}

We recall the Rademacher-type theorems of Pansu and Cheeger. Then, we discuss their applications to the problem of bi-Lipschitz nonembedding. In contrast, as an application of Theorem 3.1 we will prove that the Grushin plane equipped with Carnot-Carathéodory distance embeds bi-Lipschitzly into Euclidean space. Thus, we obtain the first example of a sub-Riemannian manifold admitting a bi-Lipschitz embedding.

We recall definitions of the Heisenberg group and the Grushin plane.

Definition 4.1. The Heisenberg group $\mathbb{H}$ is $\mathbb{R}^{3}$ with horizontal distribution spanned by two vectors

$$
X_{1}=\frac{\partial}{\partial x}-\frac{y}{2} \frac{\partial}{\partial z} \quad \text { and } \quad X_{2}=\frac{\partial}{\partial y}+\frac{x}{2} \frac{\partial}{\partial z} .
$$

It is the first non trivial example of step 2 Carnot group and it has dilations

$$
\delta_{\lambda}(x, y, z)=\left(\lambda x, \lambda y, \lambda^{2} z\right) .
$$

Definition 4.2. The Grushin plane $\mathbb{G}$ is $\mathbb{R}^{2}$ with horizontal distribution spanned by

$$
X_{1}=\frac{\partial}{\partial x} \quad \text { and } \quad X_{2}=x \frac{\partial}{\partial y} .
$$

We next define dilations $\delta_{\lambda}$ on $\mathbb{G}$ by

$$
\delta_{\lambda}(x, y)=\left(\lambda x, \lambda^{2} y\right)
$$


whenever $p=(x, y) \in \mathbb{G}$ and $\lambda>0$. Then, $X_{1}$ and $X_{2}$ are homogeneous of degree one with respect to the dilations. Hence, the Carnot-Carathéodory distance satisfies

$$
d_{c c}\left(\delta_{\lambda}(p, q)\right)=\lambda d_{c c}(p, q) .
$$

for all $p, q \in \mathbb{G}$. See (4.5) for definition of Carnot-Carathéodory distance. The points on the line $x=0$ are singular, while the other points in the plane are regular. We write $\mathbb{A}$ for the set of singular points. For more information about the Grushin plane, see $[2]$.

Remark 4.1. The Grushin plane with Carnot-Carathéodory distance is a globally doubling measure space and satisfies globally Poincaré inequality with respect to Lebesgue measure.

The Grushin plane $\mathbb{G}$ with Lebesgue measure is a locally doubling metric measure space satisfying locally $p$-Poincaré inequality for any $p \geq 1$ ([11], [14]). We fix a compact $K$ which contains a neighborhood of the origin and $r_{0}>0$. For any $p \in \mathbb{G}$ and any $r>0$, we choose $\lambda>0$ so that $\delta_{\lambda}(B(p, 2 r))=B\left(\delta_{\lambda}(p), 2 \lambda r\right)$ is contained in $K$ and $\lambda r \leq r_{0}$. Then the doubling condition holds for $\delta_{\lambda}(B(p, r))=B\left(\delta_{\lambda}(p), \lambda r\right)$ and $\delta_{\lambda}(B(p, 2 r))=B\left(\delta_{\lambda}(p), 2 \lambda r\right)$. Since $\mu\left(\delta_{\lambda}(E)\right)=\lambda^{3} \mu(E)$ for any set $E \subset \mathbb{G}$ we conclude the doubling condition for $B(p, r)$. A similar argument applies to the Poincaré inequality.

4.1. Bi-Lipschitz nonembedding theorems. In Euclidean space, Rademacher's theorem states that a Lipschitz function is differentiable almost everywhere and the derivative is linear. We shall state theorems of Pansu and Cheeger which are analogues of Rademacher's theorem in some sense. These theorems can be applied to get nonembeddability of some metric spaces into Euclidean space.

Theorem 4.1 (Pansu [21]). Let $(M, \bullet)$ and $(N, \star)$ be Carnot groups. Every Lipschitz mapping $f$ between open sets in $M$ and $N$ is differentiable almost everywhere. Moreover, the differential

$$
d f_{y}(x)=\lim _{t \rightarrow 0} \delta_{t^{-1}}\left[f(y)^{-1} \star f\left(y \bullet \delta_{t}(x)\right)\right]
$$

is a Lie group homomorphism almost everywhere.

Here $\left(\delta_{t}\right)$ denotes the family of dilations in $M$ or $N$. See (4.1) for the case of the Heisenberg group.

Semmes [23] observed that Theorem 4.1 implies that nonabelian Carnot groups $M$ cannot be embedded bi-Lipschitzly in Euclidean space. If $M$ had a bi-Lipschitz embedding $f$ into some Euclidean space $\mathbb{R}^{n}$, then $f$ must be differentiable in the sense of Pansu and its differential should be an isomorphism. This gives a contradiction because it has nontrivial kernel. Hence $M$ cannot be bi-Lipschitz embeddable. In particular, the Heisenberg group does not admit a bi-Lipschitz embedding into Euclidean space.

Rademacher's theorem states that infinitesimal behavior of any Lipschitz functions on $\mathbb{R}^{n}$ is approximated at almost every point by some linear function; that is, a linear combination of the coordinate functions. Cheeger proved a remarkable extension of Rademacher's theorem in doubling metric measure spaces supporting a $p$-Poincaré inequality. He constructed coordinate charts that span the differentials of Lipschitz 
functions. Moreover, his work gives a way to get nonembeddability results by using a purely geometric and analytic method.

Theorem 4.2 (Cheeger [4]). If $(X, d, \mu)$ is a doubling metric measure space supporting a p-Poincaré inequality for some $p \geq 1$, then $(X, d, \mu)$ has a strong measurable differentiable structure, i.e., a countable collection of coordinate patches $\left\{\left(X_{\alpha}, \pi_{\alpha}\right)\right\}$ that satisfy the following conditions:

(1) Each $X_{\alpha}$ is a measurable subset of $X$ with positive measure and the union of the $X_{\alpha}$ 's has full measure in $X$.

(2) Each $\pi_{\alpha}$ is a $N(\alpha)$-tuple of Lipschitz functions, for some $N(\alpha) \in \mathbb{N}$, where $N(\alpha)$ is bounded from above independently of $\alpha$.

(3) Given a Lipschitz function $f: X \longrightarrow \mathbb{R}$, there exists an $L^{\infty}$ function $d f^{\alpha}$ : $X_{\alpha} \longrightarrow \mathbb{R}^{N(\alpha)}$ so that

$$
\limsup _{y \rightarrow x} \frac{\left|f(y)-f(x)-d f^{\alpha}(x) \cdot\left(\pi_{\alpha}(y)-\pi_{\alpha}(x)\right)\right|}{d(x, y)}=0 \text { for } \mu-\text { a.e } x \in X_{\alpha} .
$$

Cheeger also provided a uniform statement that covers many of the known nonembedding results.

Theorem 4.3 (Cheeger). If a doubling p-Poincaré space $X$ admits a bi-Lipschitz embedding into some finite-dimensional Euclidean space, then at almost every point $x \in X_{\alpha}$, the tangent cone of $X$ at $x$ is bi-Lipschitz equivalent to $\mathbb{R}^{N(\alpha)}$.

We can deduce from Cheeger's theorem the known nonembedding results both for the Carnot groups and for Laakso spaces. Cheeger and Kleiner generalized the almost everywhere differentiability for Lipschitz maps on PI space to any Banach space $V$ with Radon-Nikodým property $([5,6])$.

We now check nonembeddability of the Heisenberg group $\mathbb{H}$ by applying Cheeger's nonembedding theorem. The Heisenberg group has a strong measurable differentiable structure with a single coordinate patch $\left(\mathbb{H}, \pi_{1}, \pi_{2}\right)$, where $\pi_{1}(x, y, t)=x$ and $\pi_{2}(x, y, t)=y$. If we assume that the Heisenberg group admits a bi-Lipschitz embedding into some Euclidean space, then every tangent cone at almost every point in $\mathbb{H}$ must be bi-Lipschitz equivalent to $\mathbb{R}^{2}$. Since the Hausdorff dimension of $\mathbb{H}$ is not equal to 2, we conclude bi-Lipschitz nonembeddability.

In contrast to the Heisenberg group, Cheeger's nonembedding theorem does not answer whether or not the Grushin plane locally embeds into some Euclidean space. The Grushin plane $\mathbb{G}$ with Lebesgue measure is a doubling metric measure space supporting $p$-Poincaré inequality for any $p \geq 1$ (see Remark 4.1). Let $K$ be any compact subset of $\mathbb{G}$ and $\mathbb{A}$ be set of singular points, $y$-axis. It has a Cheeger's coordinate patch $\left(K \backslash \mathbb{A}, \pi_{1}, \pi_{2}\right)$, where $\pi_{1}(x, y)=x$ and $\pi_{2}(x, y)=y$. Since every tangent cone to $K \backslash \mathbb{A}$ is bi-Lipschitz equivalent to $\mathbb{R}^{2}$, we cannot conclude non-embeddability of the Grushin plane, unlike the case of the Heisenberg group. Indeed, we prove that the Grushin plane admits a bi-Lipschitz embedding into some Euclidean space.

4.2. The Grushin plane. The metric on $\mathbb{G} \backslash \mathbb{A}$ is the Riemannian metric $d s^{2}$ making $X_{1}$ and $X_{2}$ into an orthonormal basis for the tangent space,

$$
d s^{2}=d x^{2}+\frac{d y^{2}}{x^{2}}
$$


The metric can be extended across $\mathbb{A}$ as the Carnot-Carathéodory distance (or $c c$-distance) by means of the length element $d s^{2}$, since the horizontal distribution satisfies the Hörmander condition.

For any horizontal curve $\gamma:[0,1] \rightarrow \mathbb{G}$, we write $\gamma(t)=(\mathrm{x}(t), \mathrm{y}(t))$ for a parameterized horizontal curve. Then, we have

$$
\operatorname{length}(\gamma)=\int_{0}^{1} \sqrt{\mathrm{x}^{\prime}(t)^{2}+\frac{\mathrm{y}^{\prime}(t)^{2}}{\mathrm{x}(t)^{2}}} d t
$$

The following proposition gives distance estimates for the Carnot-Carathéodory distance on $\mathbb{G}$. Nagel-Stein-Wainger [19] provide distance estimates for $c c$-metrics induced by more general families of vector fields satisfying the Hörmander condition.

Proposition 4.1. The Carnot-Carathéodory distance on $\mathbb{A}$ is comparable to $\sqrt{d_{E}}$. Now fix points $p=\left(x_{1}, y_{1}\right)$ and $q=\left(x_{2}, y_{2}\right)$ in $\mathbb{G} \backslash \mathbb{A}$. We have the following distance estimates:

$$
\begin{aligned}
& \frac{1}{2}\left(\left|x_{1}-x_{2}\right|+\frac{\left|y_{1}-y_{2}\right|}{\sqrt{\min \left(\left|x_{1}\right|,\left|x_{2}\right|\right)^{2}+4\left|y_{1}-y_{2}\right|}}\right) \\
& \quad \leq d_{c c}(p, q) \leq 4\left(\left|x_{1}-x_{2}\right|+\sqrt{\left|y_{1}-y_{2}\right|}\right)
\end{aligned}
$$

Proof. The first estimation of the $c c$-distance on $\mathbb{A}$ is deduced from equation (4.2). The upper bound in equation (4.6) comes from the triangle inequality. We will use equation (4.5) to get the lower bound in equation (4.6). Let $\gamma(t)=(\mathrm{x}(t), \mathrm{y}(t))$ be a parameterized horizontal curve joining $p$ to $q$ where $t \in[0,1]$. Then,

$$
(*) \operatorname{length}(\gamma) \geq\left|x_{1}-x_{2}\right| \text {. }
$$

If there exists $K$ such that $|\mathrm{x}(t)| \leq K$ for all $t \in[0,1]$, then length $(\gamma) \geq K^{-1}\left|y_{1}-y_{2}\right|$. Otherwise, there exists $t_{0} \in[0,1]$ such that $\left|\mathrm{x}\left(t_{0}\right)\right| \geq K$. Then,

$$
\operatorname{length}(\gamma) \geq \operatorname{length}(\widetilde{\gamma}) \geq \max \left\{\left|\mathrm{x}\left(t_{0}\right)-x_{1}\right|,\left|\mathrm{x}\left(t_{0}\right)-x_{2}\right|\right\} \geq K-\min \left\{\left|x_{1}\right|,\left|x_{2}\right|\right\}
$$

where $\widetilde{\gamma}$ is a subcurves of $\gamma$ joining $p$ to $\left(\mathrm{x}\left(t_{0}\right), \mathrm{y}\left(t_{0}\right)\right)$ or $q$ to $\left(\mathrm{x}\left(t_{0}\right), \mathrm{y}\left(t_{0}\right)\right)$. Then, we have the following:

$$
(* *) \operatorname{length}(\gamma) \geq \sup _{K>\max \left\{\left|x_{1}\right|,\left|x_{2}\right|\right\}} \min \left\{K-\min \left\{\left|x_{1}\right|,\left|x_{2}\right|\right\}, K^{-1}\left|y_{1}-y_{2}\right|\right\} .
$$

When we choose $K=\sqrt{\min \left(\left|x_{1}\right|,\left|x_{2}\right|\right)^{2}+4\left|y_{1}-y_{2}\right|}$ and average $(*)$ and $(* *)$, then we get distance estimates (4.6).

We next consider the lattice of points in $\mathbb{R}^{2}$ whose coordinates are integers. Then, this lattice determines a mesh $M_{0} \times M_{0}$. For each $j \in \mathbb{Z}$, consider the submesh $M_{j}=2^{-j} M_{0} \times 2^{-2 j} M_{0}$ which is set of cubes in $\mathbb{R}^{2}$ of sidelengths $2^{-j}$ and $2^{-2 j}$ respectively. From the above distance estimates, $\mathbb{G} \backslash \mathbb{A}$ has a Whitney decomposition. We recall this in the following Proposition 4.2.

Proposition 4.2. Let $\mathbb{A}$ be $y$-axis. Then its complement $\Omega=\mathbb{G} \backslash \mathbb{A}$ is the union of a sequence of cubes $Q$, whose interiors are mutually disjoint and whose diameters are approximately proportional to their distances from $\mathbb{A}$. More precisely,

(1) $\Omega=\cup_{Q \in W_{\Omega}} Q$. 
(2) Any two cubes are mutually disjoint.

(3) $\operatorname{dist}_{c c}(Q, \mathbb{A}) \leq \operatorname{diam}_{c c}(Q) \leq 8 \operatorname{dist}_{c c}(Q, \mathbb{A})$.

The Grushin plane is complete, doubling, and uniformly perfect metric space. Since cc-distance on $\mathbb{A}$ is comparable to $\sqrt{d_{E}}$, we apply Assouad's theorem. Then we have a $L$-bi-Lipschitz embedding $f$ from $\mathbb{A}$ into $\mathbb{R}^{3}$ for some $L$. If we verify the condition of uniformly Christ-local bi-Lipschitz embeddings, then we can conclude the following theorem.

Theorem 4.4. The Grushin plane equipped with Carnot-Carathéodory distance admits a bi-Lipschitz embedding into some Euclidean space.

It is enough to verify the existence of uniformly Christ-local bi-Lipschitz embeddings. In this case, $Q^{*}$ is the set of all Whitney cubes which intersect $Q$ and $Q^{* *}$ is the set of all Whitney cubes which intersect $Q^{*}$ (see Definition 2.5).

Lemma 4.1. The complement of $\mathbb{A}$ admits uniformly Christ-local bi-Lipschitz embeddings.

Proof. We observe that $Q^{* *}$ is a closed two-dimensional Riemannian manifold for each $Q$. For any two elements $Q$ and $Q^{\prime}$ in $\mathrm{W}_{\Omega}$, we have $Q^{\prime}=\Phi(Q)$ where $\Phi$ is composition of translation map $\varsigma$ with respect to $\{0\} \times \mathbb{R}$ and expansion map $\psi(x, y)=\left(2^{\left(j^{\prime}-j\right)} x, 2^{2\left(j^{\prime}-j\right)} y\right)$. Then, we have $\operatorname{diam}\left(Q^{\prime}\right)=2^{\left(j^{\prime}-j\right)} \operatorname{diam}(Q)$ from Proposition 4.2. Therefore, we can cover all $Q^{* *}$ by balls $B_{1}, B_{2}, \ldots, B_{N}$ of radius $\operatorname{diam}(Q)>0$ where $N$ is independent of $Q$. For each $i$, there exist $L$-bi-Lipschitz diffeomorphisms for some $L$

$$
\varphi_{i}: 5 B_{i} \rightarrow \varphi_{i}\left(5 B_{i}\right) \subset \mathbb{R}^{2} .
$$

Without loss of generality, we may assume that $\left|\varphi_{i}(x)\right| \geq \operatorname{diam}(Q)$ for all $i$ and $x \in$ $5 B_{i}$. let $u_{i} \in C_{0}^{\infty}\left(2 B_{i}\right)$ be such that $0 \leq u_{i} \leq 1$ and $\left.u_{i}\right|_{B_{i}}=1$, and let $v_{i} \in C_{0}^{\infty}\left(5 B_{i}\right)$ be such that $0 \leq v_{i} \leq 1$ and $\left.v_{i}\right|_{4 B_{i}}=1$. Then, we define $\varphi: X \rightarrow \mathbb{R}^{2 N} \times \mathbb{R}^{2 N}$

$$
\varphi(x):=\left(\varphi_{1}(x) u_{1}(x), \ldots, \varphi_{N}(x) u_{N}(x), \varphi_{1}(x) v_{1}(x), \ldots, \varphi_{N}(x) v_{N}(x)\right)
$$

Obviously $\varphi$ is smooth, and hence it is Lipschitz with Lipschitz constant $2 L N$. We will show that $\varphi$ is co-Lipschitz. To this end, let us assume first that $d(x, y)>3 \operatorname{diam}(Q)$. Then, there exists $i$ such that $u_{i}(x)=1$ and $v_{i}(x)=0$. Thus,

$$
\begin{aligned}
|\varphi(x)-\varphi(y)| & \geq\left|\varphi_{i}(x) u_{i}(x)-\varphi_{i}(y) u_{i}(y)\right| \\
& =\left|\varphi_{i}(x)\right| \\
& \geq \operatorname{diam}(Q) \geq \frac{1}{C\left(C_{1}, A, \delta\right)} d(x, y) .
\end{aligned}
$$

The last inequality arises from comparability of $\operatorname{diam}(Q)$ and $\operatorname{diam}\left(Q^{* *}\right)$ (see Proposition 2.2). On the other hand, if $d(x, y) \leq 3 \operatorname{diam}(Q)$, then there exists $i$ such that $v_{i}(x)=1=v_{i}(y)$. Thus,

$$
|\varphi(x)-\varphi(y)| \geq\left|\varphi_{i}(x)-\varphi_{i}(y)\right| \geq \frac{1}{L} d(x, y) .
$$

Therefore, we have uniformly local bi-Lipschitz embeddings on each $Q^{* *}$ into $\mathbb{R}^{4 N}$. The bi-Lipschitz constant and dimension of the target space are independent of $Q$. 
Theorem 4.4 can be generalized to cover other singular sub-Riemannian manifolds similar to the Grushin plane. We denote points in $\mathbb{R}^{n} \times \mathbb{R}^{l}$ by $p=(x, y)$, where $x=\left(x_{1}, x_{2}, \ldots, x_{n}\right) \in \mathbb{R}^{n}$ and $y=\left(y_{1}, y_{2}, \ldots, y_{l}\right) \in \mathbb{R}^{l}$. We let $\alpha=$ $\left(\alpha_{1}, \alpha_{2}, \ldots, \alpha_{n}\right)$ be an $n$-tuple of non-negative integers with length $|\alpha|=\sum_{i=1}^{n} \alpha_{i}$. If $x=\left(x_{1}, x_{2}, \ldots, x_{n}\right) \in \mathbb{R}^{n}$, we put $x^{\alpha}:=x_{1}^{\alpha_{1}} x_{2}^{\alpha_{2}} \cdots x_{n}^{\alpha_{n}}$.

Definition 4.3. The space of Grushin type is $\mathbb{R}^{n} \times \mathbb{R}^{l}$ for $n, l \in \mathbb{N}$ with horizontal distribution spanned by $X_{i}$ and $Y_{j}$ for $i=1,2, \ldots, n$ and $j=1,2, \ldots, l$

$$
X_{i}=\frac{\partial}{\partial x_{i}} \quad \text { and } \quad Y_{j}=x^{\alpha^{j}} \frac{\partial}{\partial y_{j}}
$$

where for each $j, \alpha^{j}$ is an $n$-tuple of non-negative integers $\alpha_{i}^{j}$ and $\left|\alpha^{j}\right|=k$.

Theorem 4.5. The space of Grushin type equipped with Carnot-Carathéodory distance admits a bi-Lipschitz embedding into some Euclidean space.

Proof. We can follow similar steps as the proof of Theorem 4.4. We omit the proof.

\section{Questions and remarks}

So far we have given a characterization of Euclidean bi-Lipschitz embeddability of uniformly perfect metric spaces supporting a doubling measure. The hypothesis in Theorem 3.1 is based on a Christ-Whitney decomposition deduced from uniform perfectness and existence of a doubling measure. We emphasize that uniform perfectness is only used for existence of a Christ-Whitney decomposition.

Question 5.1. Can the condition of uniform perfectness be weakened?

From Theorem 3.1, the dimension $M_{1}+M M_{2}+1$ of the Euclidean space depends on the bi-Lipschitz constant $L_{1}$ and the doubling constant of $\mu$. However, the number of colors $M$ is not optimal. Thus, the following question naturally arises.

Question 5.2. What is the minimal dimension of Euclidean space into which a metric space satisfying the conditions in Theorem 3.1 bi-Lipschitzly embeds?

As an application of Theorem 3.1, we have considered the Grushin space. We now can consider the space of Grushin type with extended horizontal distribution on $\mathbb{R}^{n} \times \mathbb{R}^{l}$.

Definition 5.1. The extended space of Grushin type is $\mathbb{R}^{n} \times \mathbb{R}^{l}$ for $n, l \in \mathbb{N}$ with horizontal distribution spanned by $X_{i}$ and $Y_{j}$ for $i=1,2, \ldots, n$ and $j=1,2, \ldots, l$

$$
X_{i}=\frac{\partial}{\partial x_{i}} \quad \text { and } \quad Y_{j}=x^{\alpha^{j}} \frac{\partial}{\partial y_{j}} .
$$

Remark 5.1. We emphasize that the lengths $\left|\alpha_{i}^{j}\right|$ can be distinct in Definition 4.3.

It seems that we can follow similar steps to prove embeddability. However, some of the technical details must be checked.

Conjecture 5.1. The extended space of Grushin type with Carnot-Carathéodory distance admits a bi-Lipschitz embedding into Euclidean space. 
In the case of spaces of Grushin type, horizontal distributions are good enough to have uniformly Christ-local embeddings. Therefore, the following problem naturally comes up.

Problem 5.3. Find sufficient conditions on a higher dimensional horizontal distribution in a given sub-Riemannian manifold so as to guarantee the existence of uniformly Christ-local bi-Lipschitz embeddability.

Even more generally, we meet the following problems:

Problem 5.4. Characterize Christ-local bi-Lipschitz embeddability.

If Problem 5.4 were solved, then we could characterize bi-Lipschitz embeddable metric spaces with geometric and analytic criteria. Therefore, we could determine which metric spaces admit a bi-Lipschitz embedding and we can classify up to bi-Lipschitz equivalence those metric spaces which are subsets of Euclidean space.

Problem 5.5. Find other examples of sub-Riemannian manifolds that satisfy conditions in Theorem 3.1 and hence, embed bi-Lipschitzly into Euclidean space.

\section{Acknowledgments}

This work was supported by US National Science Foundation Grant no. DMS0901620. I am grateful to my thesis adviser, Jeremy T. Tyson for suggesting the problem and for many stimulating conversations. I am also greatly indebted to John Mackay. The distinction between the W-large and the W-local co-Lipschitz conditions was suggested by Prof. Mackay when I took reading courses in Fall 2009 and in Spring 2010. I also want to thank Jang-Mei Wu, John P. D'Angelo, and Sergiy Merenkov for their careful reading and comments.

\section{References}

[1] P. Assouad, Plongements lipschitziens dans $\mathbf{R}^{n}$, Bull. Soc. Math. France, 111(4) (1983), 429-448.

[2] A. Bellaiche, The tangent space in sub-Riemannian geometry, in Sub-Riemannian geometry, volume 144 of Progr. Math., p. 1-78. Birkhäuser, Basel (1996).

[3] C. J. Bishop, An $A_{1}$ weight not comparable with any quasiconformal Jacobian, in The tradition of Ahlfors-Bers. IV, volume 432 of Contemp. Math., Amer. Math. Soc., Providence, RI, (2007), $7-18$.

[4] J. Cheeger, Differentiability of Lipschitz functions on metric measure spaces, Geom. Funct. Anal. 9(3) (1999), 428-517.

[5] J. Cheeger and B. Kleiner, Characterization of the Radon-Nikodým property in terms of inverse limits, Astérisque (321) (2008), 129-138.

[6] J. Cheeger and B. Kleiner, Differentiability of Lipschitz maps from metric measure spaces to Banach spaces with the Radon-Nikodým property, Geom. Funct. Anal. 19(4) (2009), 1017-1028.

[7] J. Cheeger and B. Kleiner, Metric differentiation, monotonicity and maps to $L^{1}$, Invent. Math. 182(2) (2010), 335-370.

[8] M. Christ, A T(b) theorem with remarks on analytic capacity and the Cauchy integral, Colloq. Math. 60/61(2) (1990), 601-628.

[9] M. X. Goemans, Semidefinite programming in combinatorial optimization, Math. Program. 79(1-3, Ser. B) (1997), 143-161.

[10] M. Gromov, Carnot-Carathéodory spaces seen from within, in Sub-Riemannian geometry, volume 144 of Progr. Math., Birkhäuser, Basel (1996), 79-323.

[11] P. Hajłasz and P. Koskela, Sobolev met Poincaré, Mem. Amer. Math. Soc. 145(688) (2000), $\mathrm{x}+101$. 
[12] J. Heinonen, Lectures on analysis on metric spaces, Universitext. Springer-Verlag, New York, (2001).

[13] J. Heinonen, Lectures on Lipschitz analysis, volume 100 of Report. University of Jyväskylä, Department of Mathematics and Statistics. University of Jyväskylä, Jyväskylä (2005).

[14] D. Jerison, The Poincaré inequality for vector fields satisfying Hörmander's condition, Duke Math. J. 53(2) (1986), 503-523.

[15] N. Linial, Squared $l_{2}$ metrics into $l_{1}$, in J. Matousek editor, Open Problems, Workshop on Discrete Metric Spaces and their Algorithmic Application, March (2002).

[16] K. Luosto, Ultrametric spaces bi-Lipschitz embeddable in $\mathbf{R}^{n}$, Fund. Math. 150(1) (1996), $25-42$.

[17] J. Luukkainen and H. Movahedi-Lankarani, Minimal bi-Lipschitz embedding dimension of ultrametric spaces, Fund. Math. 144(2) (1994), 181-193.

[18] J. Luukkainen and Eero Saksman, Every complete doubling metric space carries a doubling measure, Proc. Amer. Math. Soc. 126(2) (1998), 531-534.

[19] A. Nagel, E. M. Stein and S. Wainger, Balls and metrics defined by vector fields. I. Basic properties, Acta Math. 155(1-2) (1985), 103-147.

[20] A. Naor and J. Lee, $L_{p}$ metrics on the Heisenberg group and the Goemans-Linial conjecture, FOCS, (2006), 99-108.

[21] P. Pansu, Métriques de Carnot-Carathéodory et quasiisométries des espaces symétriques de rang un, Ann. of Math. (2), 129(1) (1989), 1-60.

[22] S. Semmes. Bi-Lipschitz mappings and strong $A_{\infty}$ weights, Ann. Acad. Sci. Fenn. Ser. A I Math. 18(2) (1993), 211-248.

[23] S. Semmes, On the nonexistence of bi-Lipschitz parameterizations and geometric problems about $A_{\infty}$-weights, Rev. Mat. Iberoamericana 12(2) (1996), 337-410.

[24] J.-M. Wu, Hausdorff dimension and doubling measures on metric spaces, Proc. Amer. Math. Soc. 126(5) (1998), 1453-1459.

Department of Mathematics, University of Illinois at Urbana Champaign, 1409 West Green Street, Urbana, IL 61801, USA

Current address: Korea Institute for Advanced study, 85 Hoegiro, Dongdaemun-gu, Seoul, 130-722, Republic of Korea

E-mail address: meshgrid80@gmail.com 